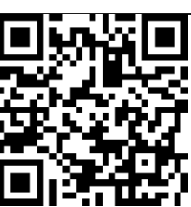

Editor's choic

Scan to access $m$

free content

${ }^{1}$ Centre for Medical Humanities, Humanities Research Institute, University of Leeds, Leeds, UK

${ }^{2}$ Research Affiliate, Program in

Placebo Studies, Harvard

Medical School, Harvard

University, Boston, USA

\section{Correspondence to}

Dr Charlotte Blease, Centre for

Medical Humanities,

Humanities Research Institute,

University of Leeds, 29-31

Clarendon Place, Leeds LS2

9JT, UK; charlotteblease@

gmail.com

Accepted 8 January 2016

Published Online First

3 February 2016

\title{
In defence of utility: the medical humanities and medical education
}

\author{
Charlotte Blease ${ }^{1,2}$
}

\section{ABSTRACT}

The idea that a study of the humanities helps to humanise doctors has become a leitmotif within the field. It is argued that the humanities (especially, literature) help to foster insights beyond those provided by biomedical training. Healthy young medics, it is claimed, can thereby gain significant insights into patienthood, and obtain important skills that may be valuable for their professional life. But the instrumentality of the humanities is not the only justification proffered for its inclusion in medical curricula. In this paper I critically examine the two overarching justifications recurrently cited in the mainstream literature-namely, (1) the instrumental worth and (2) the intrinsic value of the medical humanities in educating doctors. Examining these theses (and focusing on the views of a leading medical humanities scholar) I show that the bifurcation into instrumental versus non-instrumental justifications is not supported by the argumentation. Instead, I find that the particulars of the supposedly intrinsic justifications amount to an unambiguously instrumental defence of the humanities. Contextualizing the present investigation to probe further, I describe a long history of debate about the role of the humanities in British education and find that it rests on unsupported dichotomies (utility vs non-utility, theoretical vs applied, educated vs trained). I conclude that the medical humanities' manifesto would be more intellectually honest and coherent, and provide a more robust defence of its value in medical education, if it chose to embrace a wholly instrumental rationale for its role.

\section{INTRODUCTION}

The medical humanities often run with a mollifying manifesto. The good doctor, we are told, must also be an educated doctor. The medical humanities scholar Jane Macnaughton argues that we require doctors with "imaginative insight into the problems and contexts of patients' lives". "With these goals in mind, it is argued that the humanities afford medical students and physicians a unique window for exploring patienthood. The use of literature, in particular, is cited as an opportunity for doctors to develop sensitivity to the vast range of illness experiences and moral dilemmas faced by their patients. ${ }^{1}$ Some scholars (such as Rita Charon) have argued that fiction fosters a "narrative competence", "an ability to acknowledge, absorb, interpret and act on the stories and plights of others" and, moreover, that such a competence is essential to the practice of medicine. ${ }^{2}$ More generally, then, the assertion that the humanities (which includes but is not restricted to: English literature, theatre studies, history of art, philosophy, anthropology, social history $)^{i}$ can play an instrumental role in educating doctors is a widely held tenet. Indeed, while defining the intellectual resemblance or even shared methodology across these diverse disciplines is profoundly problematical, open debate about the role of the medical humanities has proved more tractable.

This paper analyses the debate about the educational role of the humanities within medical curricula. It critiques two standard lines of reasoning in the literature, concentrating on a leading exposition of what I take to be the received wisdom about its role within medicine (views, I argue, that lull us into false optimism). ${ }^{1}$ The conventional viewpoint proposes that there are two ways in which the humanities are relevant to medical education-first, their instrumental value, and second, the purported intrinsic value for the doctor. I contend, however, that on deeper investigation, the arguments proposed for the humanities' intrinsic value betray keenly held instrumental concerns. Extending the analysis, I show that this bifurcation (into the instrumental and non-instrumental values of education) has a distinguished history within intellectual debate in Britain. Nevertheless, I propose that it is a history of tenuous false dichotomies: utility versus non-utility, theoretical versus applied, educated versus trained. I conclude that insistence on such dichotomies may deepen the fissure between the humanities and the sciences, and squander opportunities for justifying the instrumental potential that the humanities have to offer doctors, the future of medicine-and ultimately, the patient.

\section{THE STANDARD DEFENCE OF THE MEDICAL HUMANITIES}

While there is still much open debate about the value of the medical humanities within medical education, two complementary justifications for the medical humanities are recurrently cited: (1) their instrumental value; and (2) their intrinsic value to medical education.

To begin, what is meant by instrumental values and what are they? A plethora of outcomes have been proposed as grounds for the utility of including a study of the humanities in medicine: "promoting a patient-centred approach to medical care" 3 ; "a means of producing more empathetic and effective doctors" ; "to refine and complexify their [doctors'] judgments (phronesis) in clinical situations"; "to counteract professional

${ }^{\mathrm{i}}$ The social sciences (psychology, sociology and anthropology) are often, but not always, omitted from this list. 
burnout" 6 $^{6}$ "to reduce medical hubris"3 ; "to facilitate interdisciplinary research" 3 ; "to teach the importance of evidence" foster "a critical and questioning attitude" ${ }^{8}$; to develop "the moral imagination" 13 ; "to increase understanding of the human condition" ; "to interact with patients in a perceptive manner". 8

Notice that not all of these purported advantages are educational per se: for example, 'counteracting burnout' may be said to amount to a therapeutic outcome for doctors. Some of these conjectured outcomes focus on the creation of a different intellectual culture within medicine, one that may have ramifications for research themes and influence new clinical directions within medicine. Others include the idea that the humanities will reap improvements in clinical decision-making (perhaps, in relation to appraisals about evidence-based practice). Finally (and most prominently), it is asserted that the humanities can foster improvements in doctor-patient interactions by enhancing awareness of patienthood (through augmenting levels of empathy and moral sensitivities, and by improving bedside manner). While it should be reiterated that there is still scattered disagreement about whether these educational outcomes are realised by a medical humanities education, ${ }^{9}{ }^{10}$ the foregoing provides a widely cited register of instrumental benefits that are both explicitly (and occasionally implicitly) alluded to in medical humanities and medical education literature. ${ }^{1-8} 1112$

Even more forcefully, however, scholars have argued that there is a non-instrumental rationalisation for provision of a humanities education for doctors. ${ }^{\text {ii }}$ This is robustly posed as the claim that the medical humanities "do not merely have usefulness in contributing to the development of an ends other than themselves: they also have an intrinsic value in their own right and as such are essential components of the educated mind". ${ }^{1}$ Macneill articulates the same sentiment when he expresses unease about "portrayals of the humanities and art as benign and servile in relation to medicine and the health professions", which he apprehends as the "concern that the humanities and arts are used as mere instruments to the end of producing effective practitioners". ${ }^{11} \mathrm{He}$ continues,

The allusion here to Kant's categorical imperative (that we should not use another human being 'merely as a means' to our own ends) is deliberate as it helps to isolate what it is about the instrumental justification that is troubling. The concern is that we may be treating the arts as mere instruments to effect an end... ${ }^{11}$

On this line of reasoning, it is considered that "the medical humanities are not just instrumental in creating the educated doctor;...they constitute what it means to be 'educated' as distinct from simply 'trained". ${ }^{12}$ Notice, then, that intrinsic is defined in this debate as a value that is contrasted with instrumentality-as something that does not serve a purpose for some other end.

On the face of it the distinction between instrumental and intrinsic values appears well motivated: intuitively, it may seem

\footnotetext{
${ }^{\text {ii }}$ Here the paper is restricted to the definitions of non-instrumental as they have been put forward in the medical humanities literature. There exists a wider philosophical debate on the distinction between intrinsic versus extrinsic values but this debate takes us beyond the remit of this paper. In the medical humanities, intrinsic is not co-defined with 'extrinsic' value but rather with utility. For the broader philosophical debate over the semantic distinction between intrinsic versus extrinsic values see, for example, John Dewey, Theory of Valuation, University of Chicago Press, 1939; Elizabeth Beardsley, Moral worth and moral credit, Philosophical Review 1957;66:304-28.
}

correct to speak of the utility of the humanities, and to distinguish this from what has been dubbed its intrinsic value. Yet on a closer inspection the bifurcation between instrumental and intrinsic values is not consistently maintained by medical humanities scholars nor (I will argue) is the distinction wellfounded in the context of this debate (and arguably beyond). In order to see why this is the case, it is first necessary to focus on the motivations and arguments tendered for these two modes of justification.

\section{INSTRUMENTAL VERSUS INTRINSIC VALUE: A FALSE DICHOTOMY?}

Macnaughton's defence of the role of the humanities is perhaps the most cited in the literature and provides one of the clearest and most extensive expositions for the justification of the humanities in medical education. ${ }^{1}$ Therefore, in this section I focus on the content and structure of her argumentation. Before we examine these claims, however, it is important to consider what 'intrinsic value' might mean.

Observe that if one wished to defend the idea that the medical humanities have no instrumental value-either for doctors or anyone else-such arguments must take a particular form. Downie, for example, comes close to this view when he rhetorically asks, "Can poems....and literature...help in developing ethical sensitivity?...My answer...is cautious, or even pessimistic. Literature, cannot always help, and perhaps poetry least of all the varieties of literature". ${ }^{12}$ On this line of reasoning, then, such a purported intrinsic value amounts to an appreciation of the aesthetic dimension of a work of art without eliciting any long-term changes in the perceptual capacities of the reader. Notice the tightrope one must walk in articulating any such intrinsic justifications. One could not thereby justify the reading of poetry for its own sake on the grounds that the decision to do so was based on intentions and predictions about derived enjoyment, literary appreciation, affective responses, and so on. We are thereby presented with a deceptively complicated task: for example, does the reader read the poem because she wants to feel relief or comfort? If she did so, she would be approaching the poem with her own individual, instrumental goals in mind-namely, the goal in reading the poetry would be the realisation of a desirable emotional or aesthetic response. ${ }^{\text {iii }}$ In short, the task of articulating strictly intrinsic justifications for the humanities is thorny and intricate-and arguably elusive.

Perhaps a third consideration-an alternative to the bifurcation between instrumental or intrinsic values-might be proposed. We might say that the study of the humanities has instrumental value(s) but that these provide no specific utility for medicine per se. On this view, one must be cautious to

\footnotetext{
iii Consider the following example: It might be argued that we have clear and specific instrumental goals when we read a camera instruction booklet but not when we read a great work of literature. On a closer inspection, however, the distinction does not hold. In reading a great work of literature we can certainly be said to satisfy specific instrumental ends: the intentional pleasure in intricate plots and sophisticated characters, deriving aesthetic enjoyment from the writing, or even the satisfaction of gaining insights into new, foreign, or forgotten worlds. Therefore the question is not whether these values are instrumental but whether they are instrumental for medicine or whether they promote wider (broader, or somehow less distinct) instrumental gains. I address these concerns in the final part of the paper. However, notice that if specificity is our interest, then this promotes a different definition of intrinsic value-one that is not contrasted with utility or instrumentality but one that embraces the notion of utility for some other (unspecified) ends. [I thank an anonymous reviewer for this example.]
} 
articulate what those non-instrumental-for-medicine-butotherwise-instrumental outcomes might be, and why (it may be supposed) they have no potential application to medicine.

Turning then to Macnaughton's views, she asserts that there is a strong non-instrumental value in the provision of humanities to the medical student: indeed, (as is frequently argued elsewhere) this appears to be presented as the nobler justification. It should be pointed out, at the outset, that Macnaughton does not appear to avail of the category distinction of non-instrumental-for-medicine-but-otherwise-instrumental outcomes of the humanities. Instead, she proposes that there are 'three aspects' to the non-instrumental value of the humanities: (i) education; (ii) personal development and (iii) provision of a counter-culture to medicine.

First, drawing on Downie et $a l^{7}$ and the educationalist R S Peters (1967), she contends that the humanities offer medics a distinctive kind of non-instrumental education-one that is not merely useful:

We talk of students being 'trained' to be doctors, rather than being 'educated' in medicine... Briefly, to be educated is to have a broad perspective, as distinct from the narrow focus of training. Secondly, education is a process, not a single objective. As Peters says: "to be educated is not to have arrived; it is to travel with a different view". ${ }^{1}$

She elaborates on this, arguing that "By allowing the study of literature, history or philosophy in the medical curriculum, we shall at the very least introduce breadth. But more importantly, these subjects can challenge the students... and will allow them to consider different ways of perceiving the world. This will encourage a critical and questioning attitude and help develop judgment". 1

We can certainly agree with the claim that an education helps to instil in the student an expanse of knowledge and understanding of the world; indeed Macnaughton's views echo Wilfred Sellars' definition of philosophical enquiry, “The aim of philosophy, abstractly formulated, is to understand how things in the broadest possible sense of the term hang together in the broadest possible sense of the term". ${ }^{13}$ However, we might challenge the characterisation of this justification as noninstrumental-a task that Macnaughton appears to undertake herself. She formulates very reasonable but wholly instrumental motivations for educating doctors; her argumentation is clear: an education fosters critical thinking, a questioning attitude, helps to develop judgment and allows the student to consider different ways of seeing the world. While we might choose to moderate these claims by arguing that these are certainly the aims of an education-whether an education does, in fact, instil these qualities in the learner (and if it so, to what extent) is another matter. But the central observation in respect of her views holds: the rationale is that 'an education' (which she opposes to 'training') fosters certain outcomes, and as such these are plainly instrumental goals even if they are not labelled in this way. Reflect on how this list compares to the instrumental justifications cited above ("to refine and complexify their [doctors'] judgments (phronesis) in clinical situations" ; "to counteract professional burnout" ${ }^{36}$; "to teach the importance of evidence" ${ }^{1}$; to foster "a critical and questioning attitude". ${ }^{8}$ )

The same holds for Macnaughton's second justification for the intrinsic value of the humanities: personal development. She asserts, "Education is not just concerned with what someone can $d o$, but about what kind of people they become as a result of their education". ${ }^{1}$ Expanding on this she argues that 'an education' develops a certain kind of person with a 'humane and sympathetic approach to people' and this 'is important for the good doctor'.1 Literature, she states,

will allow the students to discover their own hidden prejudices, and to challenge them. This will encourage the kind of self understanding ('fine awareness') which is essential for the development of mature human beings who are attuned and sympathetic to the perspectives and values of other people. ${ }^{1}$

Once again, far from defending the idea that the study of the humanities is valuable in itself (a notion that we have yet to make sense of $)^{\text {iv }}$ Macnaughton defines its role in terms of its purportedly tangible effects on its students: the difference it makes to their attitude, awareness, behaviour. More than this, she specifies that the study of the humanities (and in particular "plays, poems and novels") can produce more humane doctors. Observe that this justification serves to reiterate arguments that Macnaughton (and others) have presented for the instrumental benefit of the humanities in improving compassionate understanding and the conduct of doctors: many of these justifications we already mentioned (above) ("to promote a patient-centred approach to medical care" 3 ; "to produce more empathetic and effective doctors" ; to develop "the moral imagination" ; "to increase understanding of the human condition" ; "to interact with patients in a perceptive manner". ${ }^{8}$ )

Third, consider the final justification presented in support of the non-instrumental role of the humanities: its "role in providing the experience of a 'counter-culture' to medicine". ${ }^{1}$ In defence of this, she argues, medical students are hereby afforded the opportunity to counter the overweening attitude in medicine that "medical students...have an intellectual and moral superiority over other students". ${ }^{1}$ Here, again, Macnaughton does not furnish us with non-instrumental justifications-quite the opposite. We are told, "The opportunity to take a humanities subject will allow medical students to meet teachers and students in other disciplines, will help reduce this isolation and may ultimately foster better relationships between doctors and the "outside world"'. 1

Again medical humanities may afford medical students interactions with such positive pro-social outcomes for patient-doctor relationships (we might add, however, that whether this is the case, also needs to be determined), but the point at issue (for now) is that this amounts to an instrumental justification.

In summary, upon examination, none of the three arguments proffered for the intrinsic value of the humanities is different in kind from instrumental justifications for its inclusion in medical education (indeed, there is even an explicit overlap in purported utilities). To reiterate: as the debate has been framed by Macnaughton (and others) the task of justifying the intrinsic value of the medical humanities involves the claim that the study of the humanities is worthwhile even when it has no application-including making no useful difference to the students' outlook, understanding or knowledge of the world. As I have pointed it, this is a more onerous task than has been

\footnotetext{
${ }^{i v}$ When we question medical humanities as valuable for its own sake within medical education, we might reasonably query: what is this intrinsic, inestimable value with no utility? And why should medical students get more of it than anyone else? Notice that to shift the debate to the importance of a broad education is not to discuss its value for medics per se but to enter into the wider debate about quality of education for every student. Such a shift in focus is not uncommon in the medical humanities literature but it is not an argument which specifically (uniquely) belongs in a medical forum.
} 
hitherto appreciated; moreover, as we have seen, this is manifestly not what Macnaughton and other scholars have in mind in their defence of the intrinsic role of the medical humanities. The point is modest but not insignificant: care must be taken to avoid conceptual misappropriation or the misidentification of one's own argumentation. If a case can be mounted for the instrumental value of the humanities, it deserves to be made with clarity and intellectual honesty. Before elaborating on this point, it is helpful to examine the intellectual lineage of this discussion.

\section{THE TWO CULTURES CHASM}

The presentation of a manifesto for the humanities in medicine may be a relatively recent phenomenon, but it re-enacts an older (and still lively) conversation about the significance of the humanities in university education. It is important to survey the character and tone of this debate in order to understand the nature (and limitations) of the present discussion; indeed, as Collini observes it is a conversation with a peculiarly British pedigree. $^{\mathrm{v}} 14$

The case can be made that the origins of this (frequently public) debate are located in Cardinal John Henry Newman's 1852 lectures on 'The Idea of a University' (published 2 years later as an essay). ${ }^{15}$ Newman's broad conception of the university ideal was of a 'liberal education' which "made men"vi : university students should not merely be trained in some specific set of skills (the professions such as medicine, law or banking), they should be taught "to think and to reason and to compare and to discriminate and to analyse". ${ }^{15}$ For Newman, a university education-and by this he meant a classical (including a theological) education-produced, "A habit of mind...which lasts through life, of which attributes are freedom, equitableness, calmness, moderation, and wisdom..."15 Open, intellectual discussion about ideas was the cornerstone of a liberal education and its definitive outcome was the development of a philosophical, sympathetic temperament. Indeed, while Newman unequivocally heralded such a liberal education as an instrument for the intellectual and the moral cultivation of young minds, he also defended the acquisition of a classical education as "knowledge for its own sake", thereby defending it against any expected devaluation in utilities or applications. Interestingly, Newman considered such an education (largely typified by the exploration of classical texts) to be both instrumental (it 'made men') and valuable for 'its own sake'. Here we locate the contention that the content of knowledge may be described as of purely intrinsic worth: an ideal that seems puritanical and praiseworthy (arguably diaphanous).

\footnotetext{
${ }^{\mathrm{v}}$ As Collini points out, in the USA and in Continental Europe the division between science and the humanities, and the social standing of these respective fields, has not been so trenchantly dichotomised as in Britain. Collini notes, "[T]he situation in England still contrasts strikingly not only with the pattern in the United States, but also with those in other European countries, where a different inheritance of cultural attitudes as well as cultural arrangements has given a distinctive inflection to the 'two cultures' theme". ${ }^{14}$. For example, in Germany, the term Geisteswissenschaft is often translated as 'humanities' but in fact transgresses the humanities/science divide. Instead, it is closer in meaning to 'systematic knowledge' or 'human sciences'. Similarly, in the USA, undergraduate degree programmes require students to undertake a broad range of subjects (unlike their British peers). US students must include components of the humanities, social sciences and natural sciences as part of their general education undergraduate degree requirements.

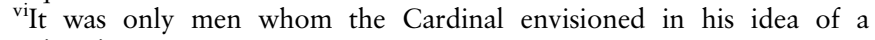
university.
In contrast to Newman's traditional ideals, mid- to late 19th century Britain witnessed the emergence of the new civic universities ('redbrick universities'), which ventured beyond traditional humanities and arts subjects to include the natural sciences, applied sciences (such as engineering) and commerce. It was also during this era that the first of two notable 'two cultures' lectures took place between poet and educator Matthew Arnold and the biologist T H Huxley ('Darwin's bulldog') (in the early 1880s). The Huxley-Arnold debate amounted to a very public squaring off on the importance of a classical education. ${ }^{\text {vii }}$ On the one hand, Huxley attacked the traditional English endorsement of a distinctively literary education with its marginalisation of the sciences. ${ }^{14}$ In response, Arnold conceded that a literary education might include all scientific classics (such as Newton's Principia and Darwin's The Origin of Species) but that it was sufficient for students to be educated in the results of such scientific enquiries rather than to acquire an understanding of how they were obtained. ${ }^{14}$ And so, the premises underlying the debate emerged: the assumptions that we might easily demarcate the humanities from the sciences; subject matter from methodology; content from critical attitude. Collini observes that the ruling, elite English education system continued to value the arts (the 'classics') above the sciences ("a vocational and slightly grubby activity, not altogether suitable for the proper education of a gentleman"). ${ }^{14}$ In this way, intellectual and public debate crystallised around the transmission of the idea that two utterly distinctive intellectual traditions existed.

The culmination of this debate in the Twentieth Century was C P Snow's 'Two Cultures', Rede lecture in 1959. Snow (a former scientist, civil servant, and bestselling novelist in his era) used the occasion of this annual, appointed public lecture at Cambridge University to present a prominent attack on what he perceived to be a pernicious, anti-intellectual attitude within British education and culture. Unlike other Western nations, he argued, British intellectual life was deeply divided: he lamented "the literary intellectuals, who incidentally while no one was looking took to referring to themselves as 'intellectuals' as if there were no others". ${ }^{16} \mathrm{He}$ argued that literary academics were shamelessly proud of their disengagement with the sciences and natural world ('natural Luddites'), basking in a "rooted impression that the scientists are shallowly optimistic, unaware of man's condition". 16

While Snow's critique was noteworthy in its own right, arguably its place in history was cemented by F R Leavis' vitriolic rejoinder. ${ }^{17}$ The celebrated literary critic was brazen (and frequently ad hominen) in his attack: snobbish (magisterial, even) and vituperously defensive. ${ }^{\text {viii }}$ Leavis admonished what he took to be Snow's caricature of literary culture and argued (ironically, in somewhat clunky style) that "for the university English School there is a creative front with which, of its function and nature, the School must be in the closest relation"; this role, he argued was located "on the contemporary intellectual-cultural frontier in maintaining the critical function....[S] uch a school would generate in the university a centre of consciousness (and

\footnotetext{
vii Collini argues that the term 'humanities' was not the predominan term in the 19th century, and 'the arts' or 'classics' was used to group together subjects such as literature, philosophy, art, ancient and modern languages, as differentiated from the study of the natural sciences, and newly emergent psychological sciences (Introduction, The Two Cultures: The Significance of C P Snow, by F R Leavis, Cambridge: Cambridge University Press, 2013).

viii Collini summarises that Leavis' response was perceived as "the crassest kind of academic imperialism" (2013).
} 
conscience) for our civilisation". ${ }^{17}$ Thus Leavis saw an instrumental role for the literary critic, and it is noteworthy that this role was cast as impervious to the knowledge and domain of the scientist.

Today's debate about the role of the medical humanities in education lacks any such invective, but its contours are no less recognisable. The justifications for the medical humanities still pivot on easily stated but not easily defended dichotomies: intrinsic worth versus evident utility; theoretical versus applied; educated versus trained. I argue that when we move past leaden rhetoric and well-worn cliché in the current debate, such bifurcations do not hold.

\section{FALLING THROUGH THE GAP: THE DANGERS OF DICHOTOMIES}

Let us return to the proposition within the medical humanities literature that knowledge can be esteemed 'for its own sake' in contradistinction to its instrumental value. As I have argued, this implies a walled protectorate around ideas, a defence of knowledge without any utility. It also amounts to the idea that we can separate content from application or utility. On further investigation, however, such knowledge is not usually lauded because it is judged to be non-instrumental. Learning is prized (whatever its subject matter) because it yields outcomes-whether in terms of the content of that knowledge, or in terms of the process of understanding that content (as Macnaughton appears to concede). Just as Newman affirmed, we might justify breadth of education because of what it enables us to do, the fresh perspectives it affords us and how it affects our judgments. There is a goal here, no less than the more narrow sense in which training is instrumental-moreover, that goal apparently has medical educational application.

The purpose of a broad education-one that encompasses the sciences and humanities-might then be justified as a means to an end and that end (it is claimed) is the cultivation of reflective, critical minds: individuals whom (it is argued) become better attuned to understand and engage in important debates about healthcare and its practice. To classify the role of the medical humanities as intrinsically valuable-where that value is defined as non-instrumental-is to miss a fundamental point. It is also, unwittingly, to embrace hobbyism: we manifestly do not celebrate the pursuit of knowledge because it is akin to train spotting or stamp collecting-pursuits which are typically characterised as intellectually, personally and socially restricted. ${ }^{\text {ix }}$

We can go further. Arguments about content versus application suggest that one can straightforwardly divest understanding of the vehicles of that understanding-namely, that one can easily differentiate propositional knowledge from the metacognitive appreciation of that knowledge (in other words, from 'insight'). That such a distinction can readily be made is a shared assumption among both the defenders and critics of the value of the medical humanities in medical education. Consider the following rhetorical comments by a medical doctor:

Shall they study ancient treatises about black bile and melancholy? For most practicing surgeons...the answer is easy. 'Let the

\footnotetext{
${ }^{\mathrm{ix}}$ Indeed, this characterisation could be criticised as unfair too: presumably these activities bring a range of social benefits and enjoyment to individuals. The point is to strike home that an education in the humanities is more appositely, coherently characterised (within the terms of the present debate) as deriving a means to another end (one that is esteemed as valuable) rather than simply justified as an end in and of itself.
}

laboratory doctors study bile salts and the humanities scholars search for meaning in the humours...but I will learn to remove stones from the common duct as safely as possible. ${ }^{10}$

Is this view justified? It is not clear that the distinction holds up. Why might learning about the humoural theory of melancholy (its content) trigger a deeper understanding of contemporary medical knowledge? One might contend that serious study in the humanities is a meta-cognitive enterprise-it is not merely the passive assimilation of a repository of facts (akin to the hobbyism of the stamp collector)-rather, it asks a variety of questions about the nature of ideas and concepts. In the case of the humoural theory, it is an invitation to enquire about the following: Why did this theory arise, and why and when (under what conditions, for example, and for what reasons) did practitioners adopt a different theory of melancholia? Is depression the same thing as melancholia? Did the humoural theory amount to science? Is contemporary psychiatry scientific? How have standards of evidence changed? Is depression a brain disease? Does it matter if patients misconceive their illness? How do different levels of analysis fit together: how does a psychological level of explanation fit with neuroscientific levels of analysis? In short, a raft of philosophical and historical questions can open up to the student upon contemplation of just one theory from the history of medicine. What instrumental utility might be derived from this content? It might be argued that the purported outcomes are more expansive than training in, for example, reading blood pressure charts or performing a tracheotomy but that these are no less instrumental and no less relevant to a professional career in medicine (whether as a clinician or as a clinician-researcher). In short, it might be stated that the humanities can afford a unique entry point into numerous contemporary and controversial theoretical issues in medicine, including such diverse issues as what counts as scientific literacy in regard to diagnosis and sources diagnostic error, debates about standards of evidence in clinical trials, as well as the ethical and legal limits of professional responsibility. As such, the humanities might be advocated as a fundamental feature of medical training: the humanities, it might be plainly asserted, help to provide doctors with a synoptic understanding of their profession, provides them with the intellectual tools to expedite and to be receptive to advancements in medicine, and to provide the expertise to engage in debates about professionalism.

It might be argued, however, that there is no evidence for any of these outcomes. Indeed, a stronger claim is that the value of the humanities cannot and should not be measured. ${ }^{18}$ Such a claim may, in part, be motivated by anxiety that the humanities cannot demonstrate their instrumental value, leading to the non sequitur that the humanities therefore have non-instrumental value. I think we must resist these moves, seductive as they may be. Just because any purported instrumental value is difficult to gauge (ie, not currently epistemically determinable-nor perhaps determinable in the foreseeable future) does not mean that such an endeavour does not make a difference to medicine. One can certainly agree that measuring such things as progress in medicine, or even differences in ethical clinical practice, are enormously challenging.

But to retreat into the recourse that the humanities are therefore intrinsically valuable-immeasurable and uniquely to be esteemed-is anti-intellectual. The notion of intrinsic value promotes academic insularity: resistance to the view that interdisciplinary work can begin to address these concerns-either now or in the future. It also promotes the idea that the humanities 
are to be respected even when instrumental gains (the rigour of meta-cognitive questioning and criticism) do not routinely occur.

Yet I take it that we do not wish to espouse the learning of facts (such as the humoural theory) without promoting in the mind of the student some reflective thinking (and thereby the potential for intellectual and analytical gains). Of course, it is certainly conceivable that the disengaged student (just as the quoted doctor, above, argues) would glean nothing about the nature of contemporary medicine from this or any other historical excursion; it is also conceivable that some teachers would fail to engage students in wider analytical questions. These are certainly issues of educational and pedagogical focus for the humanities. They also raise important normative issues about whether we truly value instrumentality in teaching the humanities to medical students. But notice that even if such meta-level questions were never raised in a medical humanities curriculum, and the content of (for example) medical history was somehow presented without any Socratic analysis or reflection, it would seem peculiar to defend the value of the humanities on the grounds that content might easily be divorced from thinking about that content (or even that it should be). The demarcation implies that (a few, possibly many) students would not thereby begin (intuitively) to question the content of contemporary medicine in light of these facts (even if they were not encouraged to do so). Furthermore, maintaining this distinction (esteeming content vs valuing instrumental outcomes) arguably makes it easier to take the view that the humanities should have no truck encouraging a critical aptitude among students.

Finally, maintaining a division between the purely theoretical, on the one hand, and the applied, on the other, is difficult to sustain. Theories determine the research questions that we ask: theories shape empirical research; indeed, meta-theoretical concerns (or assumptions) about the limitations of such research are no less relevant to practical or applied outcomes. In short, there is no non-arbitrary line between the pure and applied, the theoretical and the practicable. As Collini observes, "Human understanding, when not chained to a particular instrumental task, is restless, always pushing onwards, though not in a fixed or entirely knowable direction, and there is no one moment along that journey where one can say...that the degree of understanding being sought has passed from the useful to the useless." ${ }^{19}$ Or, as the medical educator Abraham Flexner argued in his essay "The usefulness of useless knowledge"-nearly 80 years ago, in 1939-when it comes to scientific innovation, as with other scholarly research:

\begin{abstract}
Almost every discovery has a long and precarious history....Even in the pursuit of strictly practical aims an enormous amount of apparently useless activity goes on. Out of this useless activity there come discoveries which may prove of infinitely more importance to the human mind and to the human spirit than the accomplishment of the useful ends for which [professional] schools were founded. ${ }^{20}$
\end{abstract}

It has been keenly observed that commonplace reference to 'economic returns' and measurable 'impact' are the dominant agenda among academic funding bodies. Many in the humanities have bemoaned the myopia of contemporary evaluative processes $^{21}$; but it is often forgotten that such criteria risk devaluing theoretical research wherever it occurs-in the sciences as well as the arts and humanities. In summary, there is a danger in the unreflective perpetuation of false dichotomies and in the defensive silos that they burrow. ${ }^{22}$

\section{CONCLUSIONS: THE IMPORTANCE OF CANDOUR ABOUT} UTILITY

Talk of instrumental value may be perceived as imprudent, even impudent. It invites difficult questions. But there are insidious consequences of placing the humanities on a lofty pedestal, where they can be admired but do no heavy lifting, where they are above the workmanship of application. It is a sort of conceit, a demand for respect for its own sake, Vicki Pollard style. When we take this stance we miss important, promising opportunities for justifying its continued presence within medical curricula. We also miss opportunities for intellectual humility in self-criticism and refinement of these goals.

My argument is that when we look closer, the medical humanities may be on a surer footing and more intellectually honest when they stick their head above the parapet. The hypothesis that dare not speak its name-yet is abundant within justifications for the role of the humanities in medicine-is the assertion that such disciplines can improve medicine. This is a straightforward instrumental proposition-one which is, in principle, if not in practice, evidentiary. It is time that we embraced it and the challenges that it presents. To do so would be to encourage serious debate about the ways in which the humanities have the potential to influence medical research, and clinical practice. It would also restore patienthood-enhancing the health and wellbeing of the individual-as the unembarrassed, overarching goal of medical education.

Funding Wellcome Trust, grant number 05615/Z/14/Z.

Competing interests None declared.

Provenance and peer review Not commissioned; externally peer reviewed.

\section{REFERENCES}

1 Macnaughton J. The humanities in medical education: context, outcomes and structures. Med Humanit 2000;26:23-30.

2 Charon R. Narrative medicine: a model for empathy, reflection, profession, and trust. JAMA 2001;286:1897-902.

3 Gordon J. Medical humanities: to cure sometimes, to relieve often, to comfort always. Med J Aust 2005;182:5-8.

4 Jackson M. Medical humanities in medical education. Med Educ 1996;20:395-6.

5 Shapiro J, Coulehan J, Wear D, et al. Medical humanities and their discontents: definitions, critiques, and implications. Acad Med 2009;84:192-8.

6 Wilcock SM, Daly MG, Tennant CC, et al. Burnout and psychiatric morbidity in new medical graduates. Med J Aust 2004;181:357-60.

7 Downie R, Hendry RA, Macnaughton RJ, et al. Humanizing medicine: a special study module. Med Educ 1997;31:276-80.

8 Grant VJ. Making room for medical humanities. Med Humanit 2002;28:45-8.

9 McManus IC. Humanity and the medical humanities. Lancet 1995;346:1143-5.

10 Mandell H. Humanities and medicine (a slightly dissident view). Yale J Biol Med 1992;65:183-7.

11 Macneill PU. The arts and medicine: a challenging relationship. Med Humanit 2011;37:85-90

12 Downie R. The role of literature in medical education. A commentary on the poem: Roswell, Hanger 84. J Med Ethics 1999;25:529-31.

13 Sellars W. Philosophy and the scientific image of man. In: Colodny R, ed. Frontiers of science and philosophy. Pittsburgh: University of Pittsburgh Press, 1962:35-78.

14 Collini S. Introduction. In: Snow CP, ed. The Two Cultures. Cambridge: Cambridge University Press, 1998:vii-Ixxiii.

15 Newman JH. The idea of a university. London: Aeterna Press, 1854/2015.

16 Snow CP. In: Snow CP, ed. The Two Cultures. Cambridge: Cambridge University Press, 1959/1998:4-5.

17 Leavis FR. The Two Cultures: the significance of C. P. Snow. Cambridge: Cambridge University Press, 1962/2013.

18 Macnaughton J. The dangerous practice of empathy. Lancet 2009;373:1940-1.

19 Collini S. What are universities for? London: Allen Lane, 2012.

20 Flexner A. The usefulness of useless knowledge. Harpers 1939;179:544-52.

21 Nussbaum M. Not for profit: why democracy needs the humanities. Princeton: Princeton University Press, 2012.

22 Brewer JD. The public value of the social sciences. London: Bloomsbury, 2013. 